ORIGINAL ARTICLE

\title{
Cognitive and neuroimaging findings in physically abused preschoolers
}

\author{
M R Prasad, L A Kramer, L Ewing-Cobbs
}

Arch Dis Child 2005;90:82-85. doi: 10.1136/adc.2003.045583

See end of article for authors' affiliations ....................

Correspondence to: DrM R Prasad, Department of 'Pediatrics, University of Texas Health Science Center-Houston, 7000 Fannin, Suite 2401 Houston, TX 77025, USA; mary.r.prasad@ uth.tmc.edu

Accepted 20 April 2004

\begin{abstract}
Aims: To characterise the cognitive, motor, and language skills of toddlers and preschoolers who had been physically abused and to obtain concurrent MRIs of the brain.

Methods: A between groups design was used to compare a sample of 19 children, aged 14-77 months, who had been hospitalised for physical abuse with no evidence of neurological injury to a comparison group of 19 children matched for age and socioeconomic status. Children underwent cognitive, language, and motor testing within three months of their discharge from the hospital. Caregivers of the injured children were interviewed and were asked to complete questionnaires to characterise the child's developmental level and behaviour just prior to the hospitalisation.

Results: Children who had been physically abused scored significantly lower than the comparison group on measures of cognitive functioning, motor skills, and language skills. The groups did not differ in child behaviour ratings completed by the caregivers. MRI of the brain was performed for 15 children in the physical abuse group; two were found to have significant cerebral atrophy.

Conclusions: Children who have been physically abused are at high risk for delays in cognitive, motor, and language development. Standard of care for these children should include developmental testing as well as neuroimaging of the brain to detect occult brain injury.
\end{abstract}

hild maltreatment has been shown to be related to behavioural, emotional, social, and cognitive difficulties in children. A large scale study of child maltreatment using school records and interviews found that, relative to their peers, maltreated children had significantly lower grades, repeated more grades, and had greater behavioural difficulties. ${ }^{1}$ Studies using standardised measures of cognitive functioning have also found that maltreated children perform lower than comparison children from similar sociodemographic backgrounds. ${ }^{2-4}$ Many studies of child maltreatment combined children exposed to sexual abuse, inflicted brain injury, physical abuse, and neglect into heterogeneous groups. Often the children are not screened for neurological involvement and the presence of occult inflicted brain injury in a subset of maltreated children is likely to unduly lower group performance on cognitive measures. Children who have been physically abused but do not have documented head trauma are at high risk for positive findings on neuroimaging studies. Rubin and colleagues $^{5}$ examined a sample of 65 children under the age of 2 years hospitalised for skeletal and soft tissue injuries that were high risk for causation by physical abuse. Approximately $37 \%$ of children had abnormalities on computed tomography (CT) or magnetic resonance imaging (MRI) of the brain, including skull fractures, intracranial injuries, and scalp swelling. This rate of neuroimaging abnormality is very similar to that which we identified in our studies of children with inflicted traumatic brain injury as prior abnormalities were found in $40 \%$ of the children. ${ }^{6}$

To date, no study has performed MRI of the brain and developmental testing concurrently on children who have been physically abused and have no history of head trauma. In this pilot study, we investigated the cognitive performance of preschoolers who had been physically abused and who had undergone MRI of the brain in comparison to sociodemographically matched community controls. We hypothesised that relative to the comparison group, physically abused children would perform significantly lower on measures of cognitive, motor, and language development and that their behaviour would be rated less favourably by caregivers. We also hypothesised that MRI of the brain would reveal occult brain injuries in a subset of physically abused children.

\section{METHODS}

\section{Participants}

Physical findings and developmental status were prospectively evaluated in 19 children ranging in age from 14 to 77 months who were hospitalised because of abusive injuries. No child had documented head trauma and none were reported to be sexually abused or had physical findings suggestive of sexual abuse. These children were hospitalised at Memorial Hermann Children's Hospital in Houston, Texas. Inclusion criteria were: (1) gestational age of at least 32 weeks; (2) no prior known developmental delays; (3) no pre-existing metabolic or neurological conditions (seizure disorder, cerebral palsy, etc); (4) no known history of sexual abuse; and (5) no known brain injury. Children received a detailed physical examination by a paediatrician and skeletal surveys. As part of a prospective study, MRI of the brain was performed on 15 of the children. The remaining four children could not be scanned because of sedation difficulties or parental refusal. The determination of probable abuse was made by the Child Protection Committee at each hospital and the state protective and regulatory agency (Child's Protective Services). Injuries were considered highly suspicious of abuse if there were inconsistencies between the type of injury and mechanism of injury and unexplained injuries ${ }^{78}$ (see table 1 for demographic and injury data). For children in the physical abuse group, all had bruising and lacerations consistent with intentional injury (pattern and location of bruises), 57\% sustained fractures consistent with intentional injury (spiral or multiple fractures of varying age with no history of accidental trauma), and $40 \%$ were severely burned and the pattern was consistent with abuse.

Nineteen comparison children were selected from a larger sample of 39 children with no history of injury. The 


\begin{tabular}{|ll|}
\hline Table 1 Type and location of physical abuse \\
\hline & $\begin{array}{l}\text { No. of cases } \\
n=19\end{array}$ \\
& \\
\hline Burns & 1 \\
Face & 4 \\
Hands/arms & 6 \\
Feet/legs & 3 \\
Genitalia & 1 \\
Body & \\
Fractures & 1 \\
Fibula & 1 \\
Tibia & 1 \\
Humerus & 1 \\
Clavicle & \\
Femur & 5 \\
Bruises/lacerations & \\
Hands/arms & 6 \\
Feet/legs & 3 \\
Face & 7 \\
Genitalia & 2 \\
Torso & 7 \\
Organ contusion/laceration & \\
Lungs & 1 \\
Liver & 1 \\
\hline & \\
\hline
\end{tabular}

comparison children were recruited from hospitals at which the physically abused children had been hospitalised, federally subsidised clinics, and from community notices. Exclusion criteria included: gestational age less than 32 weeks, developmental delay, and known neurological disorders. The comparison children were screened for attainment of appropriate developmental milestones prior to enrolment in the study. The comparison children were matched to the physical abuse group by age (within 4 months) and socioeconomic status (Hollingshead Four Factor Index of Socioeconomic Status ${ }^{9}$ ). The 19 children in the comparison group did not significantly differ from the physically abused children in gender $\left(\chi^{2}(1, n=38)=1.69\right.$, $\mathrm{p}=0.19$ ) (see table 2 for demographic information). Birth records were obtained from the medical facilities. If such documentation was not available, birth weight and gestational information was obtained from the biological mothers. Birth weight was missing for two comparison children and gestational age was missing for one comparison child. The groups did not differ in birth weight $(F(1,34)=1.67$, $\mathrm{p}=0.20)$ or gestational age $(\mathrm{F}(1,35)=2.43, \mathrm{p}=0.13)$. Written informed consent to participate in the study was obtained either during the initial hospitalisation or following discharge. For children under the conservatorship of the Child's Protective Services, consent to participate was obtained from the agency following placement of the child in foster care or voluntary family placement. The study was approved by and conducted in accordance with the ethical guidelines of the Institutional Review Board of the University of Texas Health Science Center at Houston.

\section{Measures \\ Cognitive and motor measures \\ General cognitive ability}

The Bayley Scales of Infant Development- $\mathrm{II}^{10}$ was employed for children ages 2-42 months of age. Bayley scores were corrected for prematurity for children with gestational ages of 32-37 weeks. Children aged 43-80 months were administered the Stanford-Binet Intelligence Scales-IV Edition. ${ }^{11}$ The Bayley Scales has a mean score of 100 and standard deviation of 15 whereas the Stanford-Binet has a mean score of 100 and a standard deviation of 16. The scores from the StanfordBinet were converted to a standard deviation of 15 , thereby placing both tasks on the same scale.
Table 2 Demographic and birth variables

\begin{tabular}{|c|c|c|c|}
\hline & \multicolumn{3}{|l|}{ Group } \\
\hline & \multicolumn{2}{|l|}{ Physical abuse } & $\begin{array}{l}\text { Normal } \\
\text { comparison }\end{array}$ \\
\hline Age at testing (mth) & & & \\
\hline$M$ & 35.25 & & 29.74 \\
\hline SD & 19.80 & & 19.89 \\
\hline$M_{\text {diff }}$ & & 5.51 & \\
\hline $\mathrm{SE}_{\text {diff }}$ & & 6.21 & \\
\hline \multicolumn{4}{|l|}{ Gestational age } \\
\hline M & 37.89 & & 39.23 \\
\hline SD & 3.39 & & 1.09 \\
\hline$M_{\text {diff }}$ & & 1.34 & \\
\hline $\mathrm{SE}_{\text {diff }}$ & & 0.83 & \\
\hline \multicolumn{4}{|l|}{ Weight at birth } \\
\hline M & 6.52 & & 7.10 \\
\hline SD & 1.66 & & 0.73 \\
\hline$M_{\text {diff }}$ & & 0.58 & \\
\hline $\mathrm{SE}_{\text {diff }}$ & & 0.42 & \\
\hline Gender (males) & 10 & & 8 \\
\hline \multicolumn{4}{|c|}{ Hollingshead Four Factor } \\
\hline I-III & 6 & & 6 \\
\hline IV-V & 13 & & 13 \\
\hline
\end{tabular}

\section{Motor skills}

The Bayley Scales of Infant Development Motor Scales-2nd Edition were used to assess motor functions in children under the age of 42 months. For children aged 43-71 months, the McCarthy Scales of Children's Abilities ${ }^{12}$ motor scales were administered. All motor scores were converted to standard scores with a mean of 100 and a standard deviation of 15.0

\section{Language}

For children aged 1-35 months, the Sequenced Inventory of Communication Development (SICD) was used..$^{13}$ Developmental age scores on the SICD were converted to developmental quotients. For children at least 36 months of age, the appropriate version of the Clinical Evaluation of Language Fundamentals (Preschool or Third Edition) ${ }^{14}$ was used.

\section{Composite developmental scores}

As there are no tests of general cognitive, language, or motor ability that span the age range in this study, we created composite scores using the age appropriate measure for each child. We have successfully used this strategy in our previous studies in this age range..$^{15}$

\section{Behavioural ratings}

\section{Adaptive behaviour}

The Vineland Adaptive Behavior Scales (VABS) were administered with the caregiver of the child to characterise the level of communication, daily living skills, socialisation, and motor functioning. ${ }^{17}$ For the physical abuse group, the caregivers were interviewed about the skills and behaviour of the child prior to the hospitalisation. The interviews were conducted within four weeks of hospitalisation.

\section{Child's behaviour}

The Parenting Stress Inventory (PSI)-3rd Edition ${ }^{18}$ is a 120 item self-report measure that assesses parental stress, child behaviour, and life stress. The variables of interest for this study are derived from the Child Domain of the PSI. The Child Domain is composed of the following subscales: Distractibility/Hyperactivity, Adaptability, Reinforces Parent, Demandingness, Mood, and Acceptability. Caregivers of physically abused children were asked to complete the PSI shortly after their child was hospitalised, on average 1.5 
$(\mathrm{SD}=1.1)$ months post-injury and to rate the child's behaviour just prior to the injury. Caregivers of the comparison children were asked to rate their child's current behaviour.

\section{RESULTS}

\section{Cognitive and motor findings}

The physical abuse group performed significantly lower than the community comparison group on measures of general cognitive functioning $(\mathrm{F}(1,36)=5.39, \mathrm{p}=0.03)$, motor skills $(\mathrm{F}(1,36)=10.66, \mathrm{p}=0.002)$, receptive language $(\mathrm{F}(1,36)=$ 9.49, $\mathrm{p}=0.004)$, and expressive language $(\mathrm{F}(1,36)=13.68$, $\mathrm{p}=0.0007)$. Table 3 presents the mean scores.

\section{Child behaviour findings}

Adaptive and child behaviour information could not be obtained on four physically abused children. Domain scores from the Vineland Adaptive Behavior Scales (VABS), were significantly lower for children who had been physically abused relative to community comparison children only on the motor scale $(\mathrm{F}(1,31)=13.03, \mathrm{p}=0.001)$. Group differences were not obtained for communication $(F(1,32)=1.11$, $\mathrm{p}=0.30)$, daily living $(\mathrm{F}(1,32)=3.46, \mathrm{p}=0.07)$, or socialisation skills $(\mathrm{F}(1,32)=2.14, \mathrm{p}=0.15)$.

Child behaviour, as ascertained from the PSI, did not differentiate the two groups. The physical abuse group was not rated as being significantly different from the community comparison group on subscales of acceptability $(\mathrm{F}(1,32)=$ $0.01, \quad p=0.92)$, adaptability $(F(1,32)=0.00, \quad p=0.96)$, demandingness $(\mathrm{F}(1,32)=2.04, \quad \mathrm{p}=0.16)$, distractibility/ hyperactivity $(\mathrm{F}(1,32)=0.04, \mathrm{p}=0.84)$, or reinforces parent $(\mathrm{F}(1,32)=1.95, \mathrm{p}=0.17)$.

\section{MRI findings}

Fifteen children in the physical abuse group underwent MRI of the brain. The scans were conducted from 49 to 482 days post-hospitalisation (median 145 days post-hospitalisation). MRI scans were reviewed by a board certified radiologist with a fellowship in MRI. The radiologist was blind to the probable cause of injury. Two children had abnormal findings on the MRI. Case 1 had mild atrophy of the frontal, temporal, and parietal regions bilaterally. Mild atrophy of the corpus callosum body and enlargement of the right lateral, left lateral, and third ventricles were also noted. Case 2 had mild frontoparietal bilateral atrophy and mild enlargement of the right and left lateral ventricles. Cases 1 and 2 had no known neurological insults. Neither child was found to have cephalohaematomas or scalp bruising at the time of hospitalisation based on physical examination. Both children were born full-term with no known prenatal or perinatal complications. Case 1 had been hospitalised with a spiral femur fracture and had bruises consistent with abuse. Case 2 was hospitalised with a spiral humerus fracture and also had bruises consistent with abuse. Both scored within the average range on cognitive measures.

\section{DISCUSSION}

This study is unique in obtaining cognitive, motor, and language testing as well as brain MRI scans in a sample of children who had been physically abused. The physical abuse group scored significantly lower than an age and sociodemographically matched group of comparison children with no history of injury on all cognitive and motor tests. On measures of general cognitive functioning, the physical abuse group scored at the 16th centile whereas the comparison children scored at the 37 th centile. These findings highlight the deleterious effects of physical abuse on the development of children. Although no child in the physical abuse group had any indication of head injury at the time of
Table 3 Developmental findings by group

\begin{tabular}{|c|c|c|c|}
\hline & Group & & \\
\hline & $\begin{array}{l}\text { Physical } \\
\text { abuse }\end{array}$ & & $\begin{array}{l}\text { Normal } \\
\text { comparison }\end{array}$ \\
\hline Cognitive & & & \\
\hline$M$ & 85.22 & & 95.82 \\
\hline SD & 18.50 & & 7.37 \\
\hline$M_{\text {diff }}$ & & 10.66 & \\
\hline $\mathrm{SE}_{\text {diff }}$ & & 4.56 & \\
\hline Motor sk & & & \\
\hline$M$ & 81.53 & & 100.47 \\
\hline SD & 19.50 & & 16.13 \\
\hline$M_{\text {diff }}$ & & 18.94 & \\
\hline $\mathrm{SE}_{\text {diff }}$ & & 5.80 & \\
\hline Expressiv & & & \\
\hline$M$ & 81.53 & & 102.63 \\
\hline SD & 20.28 & & 14.40 \\
\hline$M_{\text {diff }}$ & & 21.10 & \\
\hline $\mathrm{SE}_{\text {diff }}$ & & 5.71 & \\
\hline Receptive & & & \\
\hline M & 80.05 & & 95.58 \\
\hline SD & 17.88 & & 12.76 \\
\hline$M_{\text {diff }}$ & & 15.53 & \\
\hline $\mathrm{SE}_{\text {diff }}$ & & 5.04 & \\
\hline Adaptive & & & \\
\hline Composi & & & \\
\hline M & 83.93 & & 94.89 \\
\hline SD & 15.67 & & 13.12 \\
\hline$M_{\text {diff }}$ & & 10.96 & \\
\hline $\mathrm{SE}_{\text {diff }}$ & & 4.64 & \\
\hline Commun & & & \\
\hline M & 89.00 & & 93.74 \\
\hline SD & 17.01 & & 8.66 \\
\hline$M_{\text {diff }}$ & & 4.74 & \\
\hline $\mathrm{SE}_{\text {diff }}$ & & 4.21 & \\
\hline Daily livi & & & \\
\hline$M^{\prime}$ & 89.53 & & 99.26 \\
\hline SD & 16.79 & & 13.74 \\
\hline$M_{\text {diff }}$ & & 9.73 & \\
\hline $\mathrm{SE}_{\text {diff }}$ & & 4.79 & \\
\hline Socialisa & & & \\
\hline M & 88.67 & & 96.21 \\
\hline SD & 15.01 & & 14.86 \\
\hline$M_{\text {diff }}$ & & 7.54 & \\
\hline $\mathrm{SE}_{\text {diff }}$ & & 4.84 & \\
\hline Motor sk & & & \\
\hline$M$ & 81.60 & & 97.17 \\
\hline SD & 11.74 & & 12.81 \\
\hline$M_{\text {diff }}$ & & 15.57 & \\
\hline $\mathrm{SE}_{\text {diff }}$ & & 4.00 & \\
\hline Child be & & & \\
\hline Distractil & & & \\
\hline M & 103.45 & & 102.40 \\
\hline SD & 13.20 & & 16.35 \\
\hline$M_{\text {diff }}$ & & 1.05 & \\
\hline $\mathrm{SE}_{\text {diff }}$ & & 4.88 & \\
\hline Adaptab & & & \\
\hline$M^{\prime}$ & 105.55 & & 105.80 \\
\hline SD & 15.15 & & 16.09 \\
\hline$M_{\text {diff }}$ & & 0.25 & \\
\hline $\mathrm{SE}_{\text {diff }}$ & & 5.12 & \\
\hline Reinforce & & & \\
\hline M & 94.44 & & 101.05 \\
\hline SD & 11.98 & & 15.15 \\
\hline$M_{\text {diff }}$ & & 6.61 & \\
\hline $\mathrm{SE}_{\text {diff }}$ & & 4.51 & \\
\hline Demandi & & & \\
\hline M & 105.55 & & 98.35 \\
\hline SD & 14.10 & & 15.00 \\
\hline$M_{\text {diff }}$ & & 7.20 & \\
\hline $\mathrm{SE}_{\text {diff }}$ & & 4.73 & \\
\hline Acceptal & & & \\
\hline$M$ & 100.28 & & 99.70 \\
\hline SD & 15.90 & & 16.95 \\
\hline$M_{\text {diff }}$ & & 0.58 & \\
\hline $\mathrm{SE}_{\text {diff }}$ & & 5.37 & \\
\hline
\end{tabular}


hospitalisation, MRI of the brain revealed cerebral abnormalities in two of 15 children. Both children were found to have significant cerebral atrophy of unknown aetiology. Neither of the children's parents reported previous head trauma and both children had normal prenatal and perinatal histories. It is possible that the atrophy was secondary to occult or prior head injury caused by abusive head trauma that was not reported to medical personnel. Both children scored within the average range on measures of cognitive functioning. These two children were lost to follow up and it is not known if cognitive deficits became more evident as they aged. Our findings support Rubin and colleagues's assertion that the standard of care for children with abusive injuries should include neuroimaging of the brain in addition to skeletal surveys. The scans in the present study were not performed acutely and it is possible that acute neuroimaging may have revealed a greater incidence of cerebral injuries in this group.

Despite significantly lower performance on measures of cognitive functioning, the physical abuse group was rated as comparable to the community comparison children in communication, daily living skills, and socialisation domains. Caregiver ratings of motor behaviour were lower for the physically abused than the comparison children. Caregivers were asked about the level of the child's motor skills prior to the trauma that resulted in hospitalisation (for example, femur fracture), therefore the low motor skills should not reflect the recent bodily injuries that can decrease the child's motility. It may be that because of repeated incidents of bodily injuries, children who are physically abused have weaker motor skills. Bruising, lacerations, and other painful injuries can decrease a child's motor activity and as such decrease their ability to develop new motor skills. Interestingly, contrary to previous studies, children who had been physically abused were not rated as having worse behaviour prior to the hospitalisation than community comparison children. ${ }^{2-4}{ }^{19}$ The children in the current study are younger than those included in previous studies and it is possible that younger children display less externalising behaviours. Internalising behaviours are less reliably identified by caregivers than externalising behaviour ${ }^{20}$ and may be particularly difficult to identify in young children.

It is important that children identified as abused undergo developmental testing to document their level of functioning and to institute appropriate interventions. Abused children are at high risk for delays in their development and appropriate documentation of these deficits is essential in treatment planning and interventions. Likewise, physically abused children are also at high risk for occult head trauma and it is important that they are appropriately screened for brain injuries. Physically abused children with evidence of neurological abnormalities are likely to be at even greater risk for significant delays in their development due to the presence of both direct and indirect changes in central nervous system status resulting from injury and the deleterious effects of post-traumatic stress. ${ }^{21}$ Provision of timely and appropriate interventions may improve cognitive and behavioural outcomes of physically abused children.

\section{ACKNOWLEDGEMENTS}

Preparation of this manuscript was supported in part by the National Institute of Neurological Disorders and Stroke grant R01 NS 2946207, Accidental and Nonaccidental Pediatric Brain Injury. The assistance of the participating families and Harris County Children's Protective Services of Texas is gratefully acknowledged.

\section{Authors' affiliations}

M R Prasad, L Ewing-Cobbs, Division of Developmental Pediatrics, University of Texas Health Science Center at Houston, Texas, USA L A Kramer, Department of Radiology, University of Texas Health Science Center at Houston, Texas, USA

\section{REFERENCES}

1 Eckenrode J, Laird M, Doris J. School performance and disciplinary problems among abused and neglected children. Dev Psychol 1993;29:53-62.

2 Hoffman-Plotkin D, Twentyman CT. A multimodal assessment of behavioral and cognitive deficits in abused and neglected preschoolers. Child Dev 1984;55:794-802.

3 Rogosch FA, Cicchetti DV, Aber JL. The role of child maltreatment in early deviations in cognitive and affective processing abilities and later peer relationship problems. Dev Psychopathol 1995;7:591-609.

4 Perez CM, Widom CS. Childhood victimization and long-term intellectual and academic outcomes. Child Abuse Negl 1994;18:617-33.

5 Rubin DM, Christian CW, Bilaniuk LT, et al. Occult head injury in high-risk abused children. Pediatrics 2003;111:1382-6.

6 Ewing-Cobbs L, Kramer L, Prasad M, et al. Neuroimaging, physical, and developmental findings after inflicted and noninflicted traumatic brain injury in young children. Pediatrics 1998;102:300-7.

7 Leventhal JM, Thomas SA, Rosenfield NS, et al. Fractures in young children: distinguishing child abuse from unintentional injuries. Am J Dis Child 1993;147:92

8 Wissow LS. Child abuse and neglect. N Engl J Med 1995;332:1425-31.

9 Hollingshead A. Four factor index of social status. New Haven: Yale University Press, 1975.

10 Bayley N. Bayley Scales of Infant Development-2nd Edition. San Antonio, TX: The Psychological Corporation, 1993.

11 Thorndike RL, Hagen EP, Sattler JM. Stanford-Binet Intelligence Scale: Fourth Edition. Itasca, IL: Riverside Publishing, 1986.

12 McCarthy D. McCarthy Scales of Children's Abilities. New York: Psychological Corporation, 1972.

13 Hedrick DL, Prather EM, Tobin AR. Sequenced inventory of communication development-revised. Seattle: University of Washington Press, 1995.

14 Semel E, Wiig EH, Secord WA. Clinical evaluation of language fundamentals, third edition. San Antonio: The Psychological Corporation, 1995.

15 Ewing-Cobbs L, Kramer L, Prasad M, et al. Neuroimaging, physical, and developmental findings after inflicted and noninflicted traumatic brain injury in young children. Pediatrics 1998;102:300-7.

16 Ewing-Cobbs L, Fletcher JM, Levin HS, et al. Longitudinal neuropsychological outcome in infants and preschoolers with traumatic brain injury. $J$ Int Neuropsychol Soc 1997;3:581-91.

17 Sparrow SS, Balla DA, Cicchetti DV. Vineland Adaptive Behavior Scales. Circle Pines, MN: American Guidance Service, 1984

18 Abidin RR. Parenting Stress Index-third edition. Odessa, FL: Psychological Assessment Resources, 1995

19 Vondra JI, Barnett D, Cicchetti DV. Self-concept, motivation, and competence among preschoolers from maltreating and comparison families. Child Abuse Negl 1990;14:525-40.

20 Bloom DR, Levin HS, Ewing-Cobbs L, et al. Lifetime and novel psychiatric disorders after pediatric traumatic brain injury. J Am Acad Child Adolesc Psychiatry 2001;40:572-9.

21 Keshavan MS, Diwadkar VA, DeBellis M, et al. Development of the corpus callosum in childhood, adolescence and early adulthood. Life Sci 2002;70:1909-22. 